\title{
High-Flow Carotid Cavernous Fistula and the Use of a Microvascular Plug System: Initial Experience
}

\author{
Yamin Shwe $^{a}$ Srinivasan Paramasivam ${ }^{b}$ Santiago Ortega-Gutierrez ${ }^{b}$ \\ David Altschul $^{\mathrm{b}} \quad$ Alejandro Berenstein ${ }^{\mathrm{b}}$ Johanna T. Fifi ${ }^{\mathrm{b}}$ \\ a Department of Neurology at Mount Sinai Beth Israel Medical Center, and \\ ${ }^{b}$ Hyman-Newman Institute of Neurology and Neurosurgery, Mount Sinai Hospital, \\ New York, N.Y., USA
}

\section{Key Words}

Microvascular plug $\cdot$ Carotid cavernous fistula $\cdot$ High flow

\begin{abstract}
Purpose: We report our initial experience using a detachable microvascular plug system to occlude the internal carotid artery during endovascular treatment of high-flow carotid cavernous fistula. Case and Technique: An 87-year-old patient was admitted for acute-onset double vision with associated right-eye ptosis. Exam revealed a pupil-sparing, partial right third cranial nerve palsy. MRI showed a carotid cavernous fistula with high-flow drainage. Digital subtraction angiography showed a high-flow, right-sided, direct carotid cavernous fistula with flow from the proximal right internal carotid artery. The ophthalmic artery, posterior communicating artery and anterior communicating arteries supplied retrograde flow to the fistula through the internal carotid artery. Obliteration of the fistula was achieved through coil embolization in combination with proximal and distal microvascular plugs (Reverse Medical, Irvine, Calif., USA). Conclusion: The microvascular plug is a new addition to current endovascular embolization devices for the treatment of high-flow, direct carotid cavernous fistulas. This technique offers easy navigability through tortuous arteries, precise localization and immediate occlusion, which may allow shorter procedure and fluoroscopy times and increased cost-effectiveness. Larger case series are needed to support our observation.
\end{abstract}


Fig. 1. The MVP device (Reverse Medical) is an ovoid-shaped, selfexpanding device composed of nitinol partially covered with PTFE at the proximal portion.

\section{DOI: 10.1159/000369477}

Shwe et al.: High-Flow Carotid Cavernous Fistula and the Use of a Microvascular Plug System: Initial Experience

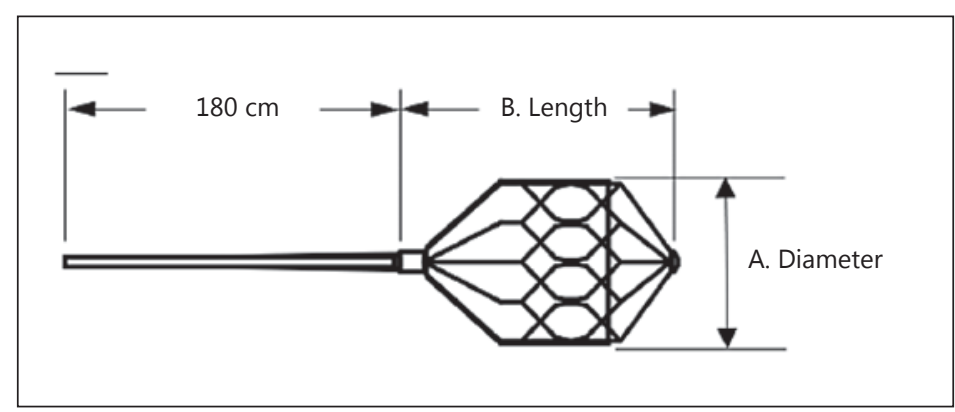

\section{Background}

Carotid cavernous fistulas (CCF) are the most common abnormal connection between the carotid artery and cavernous sinus. There are 2 types of CCF: direct and indirect. Direct CCF are high-flow fistulas occurring due to a tear in the carotid artery most commonly from either penetrating or non-penetrating head trauma [1]. Direct CCF also occur secondary to cavernous aneurysm rupture and from iatrogenic trauma following oromaxillofacial and neurosurgical procedures [2, 3]. Patients with hypertension, generalized arthrosclerosis and collagen vascular diseases such as Ehlers-Danlos syndrome tend to develop spontaneous CCF $[4,5]$. Indirect CCF occur when small dural branches of the external and internal carotid arteries (ICA) drain into the cavernous sinus.

CCF may become symptomatic immediately or may be delayed in onset up to several weeks following traumatic events depending on the drainage pathway. Anterior drainage toward the superior or inferior ophthalmic veins will lead to chemosis and exophthalmos secondary to venous congestion. Posterior drainage through the inferior petrosal vein may result in tinnitus. Cortical reflux through the sphenoparietal sinus or the superior petrosal vein could cause venous congestion, infarcts and delayed subarachnoid hemorrhage or intracerebral hemorrhage $[6,7]$.

High-flow CCF is unlikely to close spontaneously and can eventually lead to cortical reflux-related hemorrhage and blindness if left untreated [8]. The management of CCF depends on the clinical presentation and angiographic findings. Treatment involves transarterial or transvenous embolization using liquid embolics, coils and, occasionally, parent artery occlusion. For parent artery occlusion, detachable balloons have been utilized in the past, but coil embolization is most commonly used today $[9,10]$. Recently developed vascular occlusion devices, including the microvascular plug system MVP (Reverse Medical, Irvine, Calif., USA) approved by the US Food and Drug Administration in 2013 (fig. 1), have been designed for targeted vessel occlusion. We describe our experience of using the MVP in a patient with a high-flow CCF.

\section{Case}

An 87-year-old woman with a past medical history of mild dementia, diabetes, rheumatoid arthritis, and hypertension was found down at home and was brought to the hospital by EMS. The patient was presumed to have fallen.

On evaluation, she had a pupil-sparing, partial right third nerve palsy, left periorbital ecchymosis and subconjunctival hemorrhage. Initial head CT showed prominent bilateral superior ophthalmic veins without evidence of trauma or skull fracture. MR angiogram revealed features suggestive of high-flow CCF draining bilaterally with dilated cortical veins. 

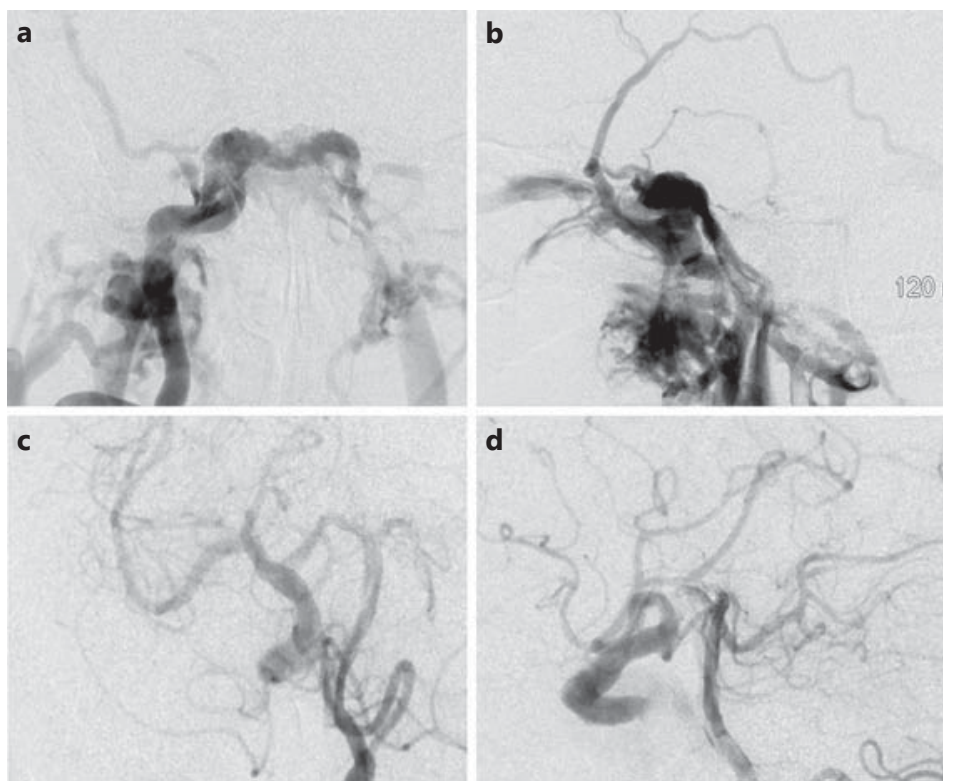

Fig. 2. a, b Posteroanterior and lateral views of a left ICA injection showing a high-flow CCF filling the cavernous sinus. There is no opacification of the distal carotid artery beyond the fistula. The venous drainage occurs medially into the contralateral cavernous sinus through the intercavernous sinus, anteriorly into the superior and inferior ophthalmic veins and laterally along the sphenoparietal sinus into the superficial middle cerebral vein and refluxing into the cortical veins. c, d Oblique and lateral views of a left vertebral injection demonstrates partial filling of the right middle cerebral artery through a large PComA that also fills the fistula in retrograde fashion through the distal ICA.

\section{Technique}

Diagnostic digital subtraction angiography (DSA) of the right ICA revealed a high-flow, direct carotid fistula filling the cavernous sinus (fig. 2a, b). The venous drainage crossed the midline to the left cavernous sinus with predominant drainage into the inferior petrosal sinus. Additional drainage into the right sphenoparietal sinus was also noted with cortical reflux into the middle cerebral vein as well as the common, superior and inferior ophthalmic veins. There was no filling of the ICA beyond the fistula with this injection. The left vertebral artery DSA showed a prominent right posterior communicating artery (PComA) filling the distal ICA and middle cerebral artery territory. Also noted was retrograde flow down the right ICA feeding the CCF (fig. 2c, d). DSA of the left ICA showed filling of the right anterior and middle cerebral artery territories through a prominent anterior communicating artery (AComA). The right cerebral hemisphere was perfused from collaterals across the circle of Willis through the AComA and PComA (fig. 2a, b).

In view of the high-flow, symptomatic CCF and angiographic evidence of cortical venous reflux with good collateral blood flow to the right hemisphere, we decided to treat the fistula by occlusion of the right ICA proximal and distal to the fistula. Vascular access into the right ICA distal to the fistula was obtained with a Reverse 027 microcatheter advanced over a Traxcess 14 microguidewire. A 6.5-mm MVP 5 was deployed in the horizontal part of the right cavernous ICA (diameter $6 \mathrm{~mm}$ ) just distal to the fistula to stop retrograde filling (fig. 3a, b). A control angiogram of the left vertebral artery showed immediate arrest of retrograde filling with stagnation of contrast in the region of the MVP. The MVP was electrolytically detached.

The microcatheter was pulled down to the vertical segment of the cavernous ICA (6.8 mm), and we attempted deployment of another 6.5-mm MVP 5. Multiple attempts were made to place the MVP in the proximal ICA, but the device was unstable due to the larger size of the parent artery. The device was resheathed, the microcatheter was advanced across the fistula, and the MVP was deployed in the right ICA at the region of the CCF. Immediate control angiogram showed a partial reduction in the flow into the fistula, and at that time, the MVP was detached. Further occlusion of the right ICA proximal to the fistula was achieved with the use of a small number of coils ( 4 coils, total length $45 \mathrm{~cm}$ ) in the proximal part of the cavernous ICA 
Fig. 3. a Lateral plain film showing deployment of the MVP (arrow) into the horizontal cavernous segment of the right ICA. b Selective lateral injection of the vertebral artery reveals immediate absence of retrograde filling of the fistula from the distal ICA and a better filling of the right middle cerebral and anterior cerebral arteries after MVP deployment.

Shwe et al.: High-Flow Carotid Cavernous Fistula and the Use of a Microvascular Plug System: Initial Experience
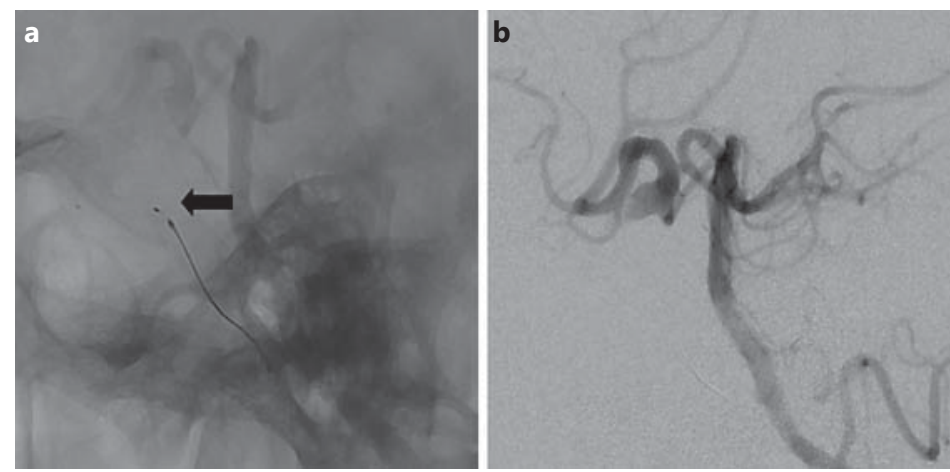

Fig. 4. a, b Plain films in posteroanterior and lateral views showing the MVP (arrow) in the ICA distal to the fistula and coil mass in the ICA proximal to the fistula. c, d DSA in lateral views of the right common carotid and left vertebral artery showing persistent complete anterograde and retrograde obliteration of the high-flow CCF.
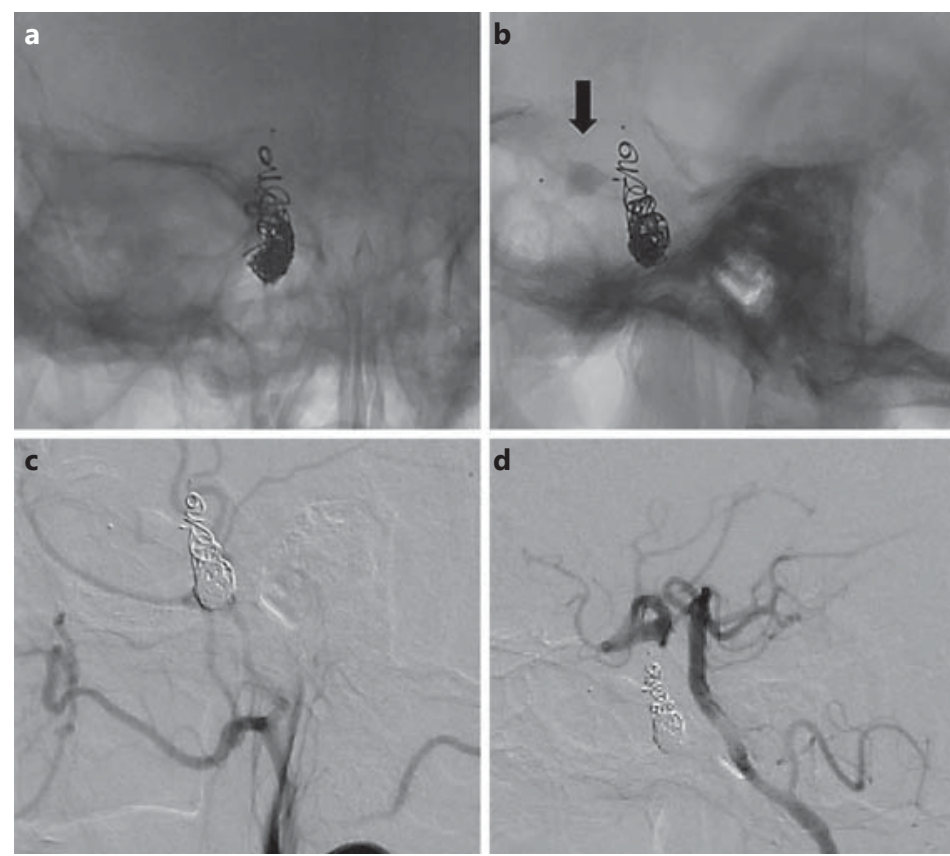

(fig. 4a, b) to ensure complete obliteration (fig. 4c, d). The collateral flow through the right PComA and AComA to the right hemisphere was confirmed using DSA of the left vertebral artery and left ICA. Antegrade flow was preserved in the ophthalmic artery. A day after the procedure, the patient's right third nerve palsy completely resolved.

\section{Discussion}

Timely intervention of high-flow CCF is critical to prevent significant morbidity. However, its management presents a number of technical challenges. Prior to the advent of endovascular therapy, treatment was performed by carotid ligation or muscle embolization through surgical exposure of the ICA [11]. Other attempted surgical options include the direct packing of the cavernous sinus and surgical disconnection of the cortical reflux [12].

Since the invention of detachable balloon catheters, the treatment of direct CCF has evolved into primary endovascular embolization. In 1974, Serbinenko [13] reported the first successful endovascular management of high-flow CCF using a transarterial flow-guided, detachable balloon. Since then, minimally invasive endovascular treatment has become the 
first-line treatment for direct CCF, primarily via transarterial or transvenous embolization using platinum coils and other embolic materials [14]. In high-flow fistulas, coil embolization on the arterial or venous side requires numerous coils, with a risk for distal migration of the coils and embolic complications with prolonged procedure time and increased expense [15].

Based on experience in peripheral and cardiac interventions, vessel occlusion devices have been used for cranial vessels $[16,17]$. Studies have shown effective embolization with the Amplatzer Vascular Plug used in conjunction with coils for the treatment of cavernous carotid aneurysm and pulmonary arteriovenous fistulas $[18,19]$. These devices are easily navigable in larger, relatively less tortuous vessels [20]. However, when used for neurointervention, these devices are limited by the small size and extreme tortuosity of the intracranial circulation. The MVP was originally used in peripheral intervention and has now been redesigned for cranial vessel occlusion [21-23]. It is an ovoid-shaped, self-expanding device made of nitinol with a partial cover of polytetrafluoroethylene (PTFE), a thin membrane causing immediate cessation of flow. PTFE is commonly used in peripheral vascular stents to reduce stent restenosis $[24,25]$. The MVP has platinum marker bands at both ends and is fully retractable prior to final deployment, allowing for more precise deployment than conventional coils. The device has good radial force and anchors itself in the wall of the blood vessels, preventing migration and immediately restricting the anterograde blood flow. The MVP is now designed to be more flexible and maneuverable through 0.027 -inch microcatheters (fig. 1). This device enables rapid, precise deployment in target vessels, potentially leading to shorter procedural time and lower cost, since it reduces the need for multiple coils when occluding high-flow fistula. In peripheral embolization, the use of a vascular plug has shown to be significantly associated with decreased fluoroscopy time and radiation compared to conventional coiling [22]. The MVP has recently been used successfully in 8 patients with various neurovascular conditions. At follow-up, there was no device migration or recanalization in 4 patients [26].

MVP placement and complete flow cessation was confirmed via a single contrast injection immediately following device deployment. In contrast, the average number and length of coils used for complete direct CCF occlusion was 14 coils (range 2-31) and a total length of $189 \mathrm{~cm}$ (range 16-736 cm) [21]. In addition, repeated contrast injection is required with coiling to access the degree of occlusion.

In our case, the first MVP device was placed in the ICA distal to the fistula with immediate occlusion of retrograde filling of the carotid fistula in less than 5 min from placement of the MVP to detachment. Due to current restrictions of MVP sizes, the second MVP was deployed at the fistula location with partial reduction in flow, and total occlusion was achieved with minimal coiling. As the cost of one MVP approximately equals the cost of one coil, we achieved cost-effective, relatively fast occlusion and successful treatment of the CCF.

However, there are certain limitations for the MVP device. First, an appropriate device selection is always needed on a case-by-case basis, keeping in mind that MVP size is currently limited. Additional MVP devices with larger diameters would be necessary to embolize larger arteries. Second, the MVP is a self-expanding device. Deployment at the CCF region in this case runs the risk of potential cranial nerve palsies and the risk of stent migration especially in cases of large CCF. In addition, parent artery occlusion increases the risk of thromboembolic complications. Third, our procedure time from groin puncture was approximately $2.5 \mathrm{~h}$, since this was our initial use of the device. We expect to have much faster procedural time with repeated use. Fourth, we do not have the follow-up angiogram of the MVP placement. However, Pellerin et al. [23] reported permanent peripheral arterial occlusion with an average follow-up of 22 days (range 22-42 days) in all 14 patients treated with the MVP. No device displacement was observed in the follow-up period. More studies 
Shwe et al.: High-Flow Carotid Cavernous Fistula and the Use of a Microvascular Plug System: Initial Experience

are needed to investigate durability and long-term migration risk. Lastly, operators must be aware that the device is not radio-opaque except for the markers at the tips, therefore introduction of radio-opaque core struts is necessary for optimal visualization during deployment.

\section{Conclusion}

Symptomatic, high-flow, direct CCF require early and effective treatment to prevent significant morbidity and mortality. The MVP is a new addition to the line of endovascular embolization devices for the treatment of high-flow CCF, offering easy navigability through tortuous arteries, precise localization and immediate occlusion, which may allow shorter procedure and fluoroscopy times and increased cost-effectiveness. Larger case series and further research comparing the MVP with other methods of treating CCF are needed to confirm our observation.

\section{References}

1 Yanoff M, Duker JS: Ophthalmology, ed 2. St. Louis, Mosby, 2004.

$>2$ Nocini P, et al: Cavernous sinus-carotid fistula: a complication of maxillofacial injury. Int J Oral Maxillofac Surg 1995;24:276-278.

3 Neil MR: Carotid-cavernous fistulas. Walsh and Hoyt's Clinical Neuro-Ophthalmology. 6th ed. Chapter 42. Lippincott Williams \& Wilkins, 2005. pp 2263-2296.

4 Rwiza HT, et al: Bilateral spontaneous carotid-cavernous fistulas, associated with systemic hypertension and generalised arteriosclerosis: a case report. J Neurol Neurosurg Psychiatry 1988;51:1003-1005.

5 Das JK, et al: Clinical spectrum of spontaneous carotid-cavernous fistula. Indian J Ophthalmol 2007;55:310 312.

6 Hayashi K, Suyama K, Nagata I: Traumatic carotid cavernous fistula complicated with intracerebral hemorrhage: case report. Neurol Med Chir (Tokyo) 2011;51:214-216.

7 Miyamoto N, et al: Clinical and angiographic characteristics of cavernous sinus dural arteriovenous fistulas manifesting as venous infarction and/or intracranial hemorrhage. Neuroradiology 2009;51:53-60.

8 Razeghinejad MR, Tehrani MJ: Sudden onset and blinding spontaneous direct carotid-cavernous fistula. J Ophthalmic Vis Res 2011;6:50-53.

9 Wu Z, et al: Treatment of traumatic carotid-cavernous fistula. Interv Neuroradiol 2000;6:277-289.

$>10$ Korkmazer B, et al: Endovascular treatment of carotid cavernous sinus fistula: a systematic review. World J Radiol 2013;5:143-155.

11 Hamby WB: Carotid-cavernous fistula. Report of 32 surgically treated cases and suggestions for definitive operation. J Neurosurg 1964;21:859-866.

12 van Dijk JM, et al: Selective disconnection of cortical venous reflux as treatment for cranial dural arteriovenous fistulas. J Neurosurg 2004;101:31-35.

13 Serbinenko FA: Balloon catheterization and occlusion of major cerebral vessels. J Neurosurg 1974;41:125145.

14 Gupta AK, et al: Endovascular treatment of direct carotid cavernous fistulae: a pictorial review. Neuroradiology 2006;48:831-839.

15 Ryer EJ, et al: Comparison of outcomes with coils versus vascular plug embolization of the internal iliac artery for endovascular aortoiliac aneurysm repair. J Vasc Surg 2012;56:1239-1245.

16 Ferro C, et al: The Amplatzer vascular plug 4: preliminary experience. Cardiovasc Intervent Radiol 2010;33: 844-848.

17 Yildiz AE, Peynircioglu B, Cil BE: Applications of the Amplatzer Vascular Plug 4. Diagn Interv Radiol 2012;18: 225-230.

18 Scott DA, et al: Vascular plug for ICA occlusion in cavernous carotid aneurysms: technical note. Neuroradiology 2008;50:795-798.

19 Abdel Aal AK, et al: Occlusion time for Amplatzer vascular plug in the management of pulmonary arteriovenous malformations. AJR Am J Roentgenol 2009;192:793-799.

20 Wang W, et al: The amplatzer vascular plug: a review of the device and its clinical applications. Cardiovasc Intervent Radiol 2012;35:725-740.

21 Luo CB, et al: Transarterial detachable coil embolization of direct carotid-cavernous fistula: immediate and long-term outcomes. J Chin Med Assoc 2013;76:31-36. 
Shwe et al.: High-Flow Carotid Cavernous Fistula and the Use of a Microvascular Plug System: Initial Experience

22 Pellerin 0, et al: Embolization of the internal iliac artery: cost-effectiveness of two different techniques. Cardiovasc Intervent Radiol 2008;31:1088-1093.

23 Pellerin 0, et al: Microvascular plug: a new embolic material for hepatic arterial skeletonization. Cardiovasc Intervent Radiol 2014;37:1597-1601.

24 Baldus S, et al: Membrane-covered stents: a new treatment strategy for saphenous vein graft lesions. Catheter Cardiovasc Interv 2001;53:1-4.

25 Schachinger V, et al: A randomized trial of polytetrafluoroethylene-membrane-covered stents compared with conventional stents in aortocoronary saphenous vein grafts. J Am Coll Cardiol 2003;42:1360-1369.

-26 Gandhi D, et al: 0-027 micro vascular plug (MVP) assisted vessel occlusion in neurovascular pathologies: technical results and initial clinical experience. J Neurointerv Surg 2014;6(suppl 1):A14. 